\title{
Pediatric cardiopulmonary arrest in the postanesthesia care unit: analysis of data from the American Heart Association Get With The Guidelines ${ }^{\circledR}$-Resuscitation registry
}

Robert Christensen, Terri Voepel-Lewis, Ian Lewis, Satya Krishna Ramachandran, Shobha Malviya for the American Heart Association's Get With The Guidelines ${ }^{\circledR}$-Resuscitation (formerly the National Registry of Cardiopulmonary Resuscitation) investigators ${ }^{a}$

Department of Anesthesiology, University of Michigan Health System, Ann Arbor, USA

\begin{abstract}
Keywords
PACU; complications; complications;

cardiac arrest; cardiac; resuscitation

\section{Correspondence \\ Robert Christensen, University of Michigan Health System, 4-911 CS Mott Children's Hospital, 1540 E. Medical Center Dr., Ann Arbor, MI 48109, USA \\ Email: robertec@med.umich.edu}

Section Editor: Andrew Davidson

Accepted 15 February 2013

doi:10.1111/pan.12154

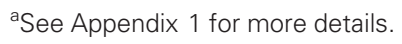

\begin{abstract}
Summary
Background: Nearly $20 \%$ of anesthesia-related pediatric cardiopulmonary arrests (CPAs) occur during emergence or recovery. The aims of this study were to describe (i) the nature of pediatric postanesthesia care unit (PACU) CPA and subsequent outcomes and (ii) factors associated with mortality.

Methods: Cardiopulmonary Arrests occurring in PACU in children ( $<18$ years) were identified from the American Heart Association Get With The Guidelines ${ }^{\circledR}$-Resuscitation, multicenter CPA registry. Demographics, underlying conditions, cause(s) of CPA, monitoring, interventions and outcomes were extracted. Descriptive statistics were used to characterize data, and odds ratios (OR) with confidence intervals $(\mathrm{CI})$ were calculated as appropriate to compare survivors and nonsurvivors.

Results: Twenty seven CPA events were included: $67 \%$ in children $<5$ years and $30 \%$ in infants $(<1$ year). Most children $(78 \%)$ had underlying comorbidities, including $15 \%$ with congenital heart disease. Respiratory issues were the most frequent causes of CPA (44\%), but cardiac/hemodynamic causes were associated with nonsurvival $(P=0.01)$. Nonsurvival was also associated with older age $(P=0.02)$, weekend occurrence $(P<0.01)$, nonpediatric setting $(P=0.02)$ and occurrence at night $(P=0.04)$.

Conclusions: This study identified similar risk factors and underlying causes as described in previous reports of pediatric perioperative CPA, with higher mortality following a cardiac/hemodynamic cause.
\end{abstract}

\section{Introduction}

Patients recovering from anesthesia are at risk for serious physiologic compromise due to proximity to the surgical procedure, residual effects of anesthetic and analgesic medications, and variable need for intensive care. Pediatric cardiopulmonary arrests (CPAs) in the perioperative period are reportedly rare and nearly $20 \%$ of such arrests occur during emergence or recovery from anesthesia (1-3). Yet, very little is known about the nature and outcomes of such events that may occur during the postanesthesia care unit (PACU) stay, where resources may be more limited compared with the operating room setting. Data regarding the nature and man- agement of pediatric PACU CPA and factors associated with favorable outcomes are needed to inform research and educational efforts with an overarching goal to improve management and outcomes following these rare but critical events.

The aim of this study was to describe the nature of pediatric PACU CPA events and subsequent outcomes using data from the American Heart Association's (AHA) Get With The Guidelines ${ }^{\circledR}$-Resuscitation (formerly, the National Registry of Cardiopulmonary Resuscitation), an international, multisite, voluntary registry of in-hospital CPA. A secondary aim was to describe factors associated with survival following pediatric PACU CPA. 


\section{Subjects and methods}

Get With The Guidelines ${ }^{\circledR}$-Resuscitation was established by the AHA in 1999 with a primary aim to evaluate CPA event data, to develop and improve evidence-based guidelines, and to improve patient outcomes. Additionally, the program provides participating institutions with feedback on their practice and outcomes to improve practices. Because the primary purpose of this registry is quality improvement, and all data are de-identified, participating hospitals are not required to obtain approval from their institutional review boards or written informed consent prior to participation. Given the de-identified nature of this retrospective database, this study met exemption criteria of the institutional review board at the University of Michigan.

The data collection methods, definitions of variables, reliability checks of data integrity and protections of patient privacy employed by the AHA in this registry have been described in multiple publications $(4,5)$ with the most recent information available at the AHA website. ${ }^{\mathrm{b}}$ To briefly summarize, designated data coordinators at participating institutions are certified by the AHA to abstract data from the medical records of patients who suffered CPA, defined as either pulselessness or a pulse with inadequate perfusion requiring chest compressions and/or defibrillation. The following data including the widely accepted Utstein cardiac arrest data elements $(6,7)$ are entered electronically with multiple checks to assist data entry and accuracy: patient demographics, pre-event status and information (e.g., comorbid conditions), monitoring and management during the event, and immediate and hospital outcomes as well as process-related quality improvement data. De-identified patient records and associated worksheets are provided to the AHA for a random sample of $10 \%$ of cases to ensure reliability. The previously reported error rate was $<3 \%$ (8). Descriptive data regarding the facilities are collected via electronic questionnaire, including self-identification as a 'pediatric only facility'. The AHA oversees training of participating sites, reliability review, and data management, as well as release of data to investigators through its national center staff, executive database steering committee and scientific advisory board. As of 2010, 1490 hospitals participated in the registry, with more than 2.3 million events in the database.

For the purpose of this study, the database was queried on 5/18/10 to obtain all CPA events that occurred in children $(<18$ years of age) in the PACU setting. Data

\footnotetext{
${ }^{b}$ Available at: www.heart.org. Accessed 5-7-12.
}

extracted included demographic data, pre-CPA conditions, monitoring, interventions, reported causes of CPA, management and outcomes, including immediate survival and status at discharge. Data were analyzed using PASW ${ }^{\circledR}$ version 18 Software (formerly SPSS, Chicago, IL). Data are described as $n(\%)$ or mean \pm standard deviation as appropriate. Chi-square, Student's t-test and Mann-Whitney U-test were used as appropriate to compare various factors between survivors and nonsurvivors. Odds ratios were computed for all relevant comparisons. $P$ values of $<0.05$ were considered statistically significant.

\section{Results}

The data query yielded 29 cases of CPA in children in the PACU setting. Following review, one newborn that arrested in the obstetric PACU was excluded, because this was not the setting of interest. Another patient had two reported CPA events during the same PACU stay, and therefore, only data from the first event were included. Data analysis included the remaining 27 cases.

\section{Pre-event information}

Pediatric postanesthesia care unit (PACU) CPAs occurred in children aged birth to 17 years with 18 events $(67 \%)$ occurring in children $<5$ years of age and 8 $(30 \%)$ in infants $<1$ year. A detailed description of the sample and a comparison of child-related factors between survivors of the CPA event and nonsurvivors is presented in Table 1.

Two children had been discharged from a PACU and another from an emergency department within $24 \mathrm{~h}$ prior to the event. All arrests were witnessed. Table 2 describes the environmental and pre-event interventional factors between survivors and nonsurvivors.

\section{Reported causes of and interventions during CPA event}

The reported cause of the CPA event was respiratory in 12 cases $(44.4 \%)$, which included nine cases reporting acute respiratory insufficiency, one inadequate invasive airway, two inadequate natural airways, one invasive airway displacement, one acute pneumothorax, and one acute pulmonary edema. A cardiac/hemodynamic cause was reported in six cases $(22.2 \%)$ which included one active myocardial infarction, four arrhythmias, and four hypotension/hypoperfusion. Mixed causes were reported in the balance of cases.

The relationship between event data and survival in the overall sample is shown in Table 3. In six cases, three attributed to respiratory insufficiency and three to 
Table 1 Prearrest conditions (mean \pm standard deviation or range, or $n(\%)$ )

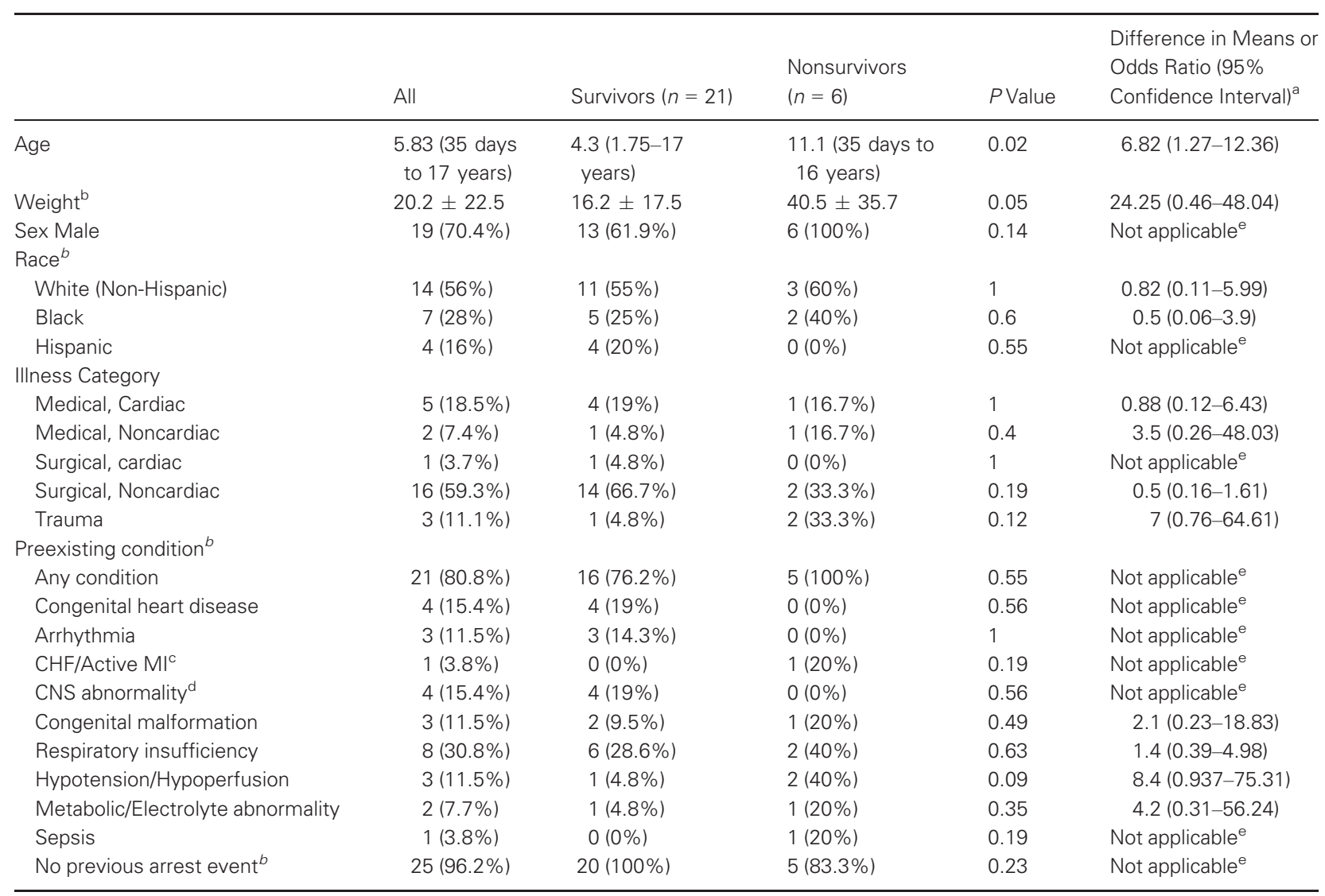

${ }^{a}$ Comparisons are made between survivors and nonsurvivors.

b Variables with missing data; percentages calculated from number of reported cases in the group.

${ }^{\mathrm{c}}$ Combines acute and chronic congestive heart failure (CHF) with active myocardial infarction (MI).

${ }^{d}$ Combines acute nonstroke event, acute stroke, and baseline depression in central nervous system (CNS) function.

e Odds ratio could not be calculated.

Table 2 Environmental factors and pre-event interventions $(n(\%)$ and odds ratio (Confidence interval))

\begin{tabular}{|c|c|c|c|c|c|}
\hline & All & Survived Event ( $n=21$ ) & Nonsurvivors $(n=6)$ & $P$ Value & Odds Ratio (Confidence Interval) \\
\hline Mixed facility ${ }^{b}$ & $15(55.6 \%)$ & $9(42.9 \%)$ & $6(100 \%)$ & 0.02 & Not applicable ${ }^{c}$ \\
\hline Weekend & $5(18.5 \%)$ & $1(4.8 \%)$ & $4(66.7 \%)$ & $<0.01$ & $14.0(1.91-102.90)$ \\
\hline Night time & $2(7.4 \%)$ & $0(0 \%)$ & $2(33.3 \%)$ & 0.04 & Not applicable ${ }^{c}$ \\
\hline Arrest Before 2005 & $11(40.7 \%)$ & $7(33.3 \%)$ & $4(66.6 \%)$ & 0.16 & $0.25(0.04-1.71)$ \\
\hline Inpatient & $22(81.5 \%)$ & $16(76.2 \%)$ & $6(100 \%)$ & 0.56 & Not applicable ${ }^{c}$ \\
\hline \multicolumn{6}{|l|}{ Monitoring } \\
\hline Pulse oximeter & $24(88.9 \%)$ & $19(90.5 \%)$ & $5(83.3 \%)$ & 0.55 & $0.92(0.63-1.35)$ \\
\hline Electrocardiography & $24(88.9 \%)$ & $19(90.5 \%)$ & $5(83.3 \%)$ & 0.55 & $0.92(0.63-1.35)$ \\
\hline \multicolumn{6}{|c|}{ Interventions in place pre-event } \\
\hline Invasive airway & $15(55.6 \%)$ & $9(42.9 \%)$ & $6(100 \%)$ & 0.02 & Not applicable ${ }^{c}$ \\
\hline Supplemental oxygen $^{d}$ & $5(18.5 \%)$ & $5(23.8 \%)$ & $0(0 \%)$ & 0.55 & Not applicable ${ }^{c}$ \\
\hline Intravascular access & $13(48.1 \%)$ & $12(57.1 \%)$ & $1(16.7 \%)$ & 0.17 & $0.29(0.05-1.81)$ \\
\hline
\end{tabular}

${ }^{a}$ Comparisons are made to all cases without cause, condition, or intervention.

${ }^{\mathrm{b}}$ Mixed facility = adult and pediatric combined setting.

${ }^{\mathrm{c}}$ Odds ratio could not be calculated.

Includes nasal cannula, face mask, and other noninvasive supplemental oxygen. 
Table 3 Cardiopulmonary arrest (CPA) event data (mean \pm standard deviation, $n(\%)$, and odds ratio (Confidence interval))

\begin{tabular}{|c|c|c|c|c|c|}
\hline & All & Survivors $(n=21)$ & Nonsurvivors $(n=6)$ & $P$ Value & $\begin{array}{l}\text { Difference in means or } \\
\text { odds ratio ( } 95 \% \text { confidence } \\
\text { interval) }^{\mathrm{a}}\end{array}$ \\
\hline \multicolumn{6}{|l|}{ Patient condition at initiation } \\
\hline Pulseless on initiation & $11(40.7 \%)$ & $8(38.1 \%)$ & $3(50 \%)$ & 0.66 & $1.31(0.5-3.46)$ \\
\hline Ventricular fibrillation & $4(14.8 \%)$ & $3(14.3 \%)$ & $1(16.7 \%)$ & 1 & $1.17(0.15-9.28)$ \\
\hline \multicolumn{6}{|l|}{ Reported causes of arrest } \\
\hline Respiratory ${ }^{\mathrm{b}}$ & $12(44.4 \%)$ & $11(52.4 \%)$ & $1(16.7 \%)$ & 0.18 & $0.32(0.05-1.99)$ \\
\hline Cardiac/hemodynamic ${ }^{c}$ & $6(22.2 \%)$ & $2(9.5 \%)$ & $4(66.7 \%)$ & 0.01 & $7.0(1.67-29.38)$ \\
\hline Cardio-respiratory mixed $^{d}$ & $8(29.6 \%)$ & $8(38.1 \%)$ & $0(0 \%)$ & 0.14 & Not applicable ${ }^{f}$ \\
\hline Other $r^{\mathrm{e}}$ & $1(3.7 \%)$ & $0(0 \%)$ & $1(16.7 \%)$ & 0.22 & Not applicable ${ }^{f}$ \\
\hline \multicolumn{6}{|l|}{ Interventions during CPA event } \\
\hline Activated hospital team & $9(33.3 \%)$ & $8(38.1 \%)$ & $1(16.7 \%)$ & 0.63 & $0.44(0.07-2.84)$ \\
\hline Invasive airway placed & $6(22.2 \%)$ & $6(28.6 \%)$ & $0(0 \%)$ & 0.28 & Not applicable ${ }^{f}$ \\
\hline Ventilated via invasive airway & $21(77.8 \%)$ & $15(71.4 \%)$ & $6(100 \%)$ & 0.28 & Not applicable ${ }^{f}$ \\
\hline Epinephrine bolus & $15(55.6 \%)$ & $9(42.3 \%)$ & $6(100 \%)$ & 0.02 & Not applicable ${ }^{f}$ \\
\hline Atropine & $15(55.6 \%)$ & $11(52.4 \%)$ & $4(66.7 \%)$ & 0.66 & $1.27(0.63-2.56)$ \\
\hline Antiarrhythmic agent & $5(18.5 \%)$ & $3(14.3 \%)$ & $2(33.3 \%)$ & 0.30 & $2.33(0.5-10.91)$ \\
\hline Reversal agent ${ }^{g}$ & $2(7.4 \%)$ & $2(9.5 \%)$ & $0(0 \%)$ & 1 & Not applicable ${ }^{f}$ \\
\hline Defibrillation & $4(14.8 \%)$ & $3(14.3 \%)$ & $1(16.7 \%)$ & 1 & $1.17(0.15-9.18)$ \\
\hline Event duration (min) & $22.5 \pm 27.7$ & $18.3 \pm 28.1$ & $36.7 \pm 22.6$ & 0.16 & $18.42(-7.56-44.42)$ \\
\hline Event to compression (min) & $0.7 \pm 0.4$ & $0.2 \pm .4$ & $0 \pm 0$ & 0.6 & $-0.1(-0.47-0.28)$ \\
\hline
\end{tabular}

${ }^{a}$ Comparisons are made between survivors and nonsurvivors.

bIncludes cases reporting one or more of the following: respiratory insufficiency, inadequate invasive airway, inadequate natural airway, invasive airway displacement, acute pneumothorax, and acute pulmonary edema.

Includes cases reporting one or more of the following: active myocardial infarction, arrhythmia, and hypotension/hypoperfusion.

${ }^{d}$ Includes cases reporting either acute respiratory insufficiency or inadequate invasive airway and either arrhythmia or hypotension/hypoperfusion.

e Includes one case reporting hypotension/hypoperfusion, conscious sedation, and metabolic/electrolyte abnormality.

${ }^{f}$ Odds ratio could not be calculated.

gSuch as naloxone or flumazenil.

arrhythmia with or without hypotension, no invasive airway was placed. Of these six cases, no medication interventions were used in 3, atropine only was given in 2 , and in the last case, glycopyrrolate only was given. All of these six children survived and were discharged home within 2.4 days of the event. The final outcomes and discharge disposition among these survivors are summarized in Table 4.

\section{Discussion}

This study describes the pediatric PACU CPA events as reported to the AHA Get With The Guidelines ${ }^{\circledR}$-Resuscitation registry and provides a preliminary, exploratory analysis of factors associated with survival from these rare events. Importantly, similar to previous studies of CPA in the perioperative period $(1,9)$, the majority of children $(63 \%)$ in this sample survived and were discharged home following the event and associated hospi-
Table 4 Description of outcomes among arrest survivors $(n=21)$

\begin{tabular}{lr}
\hline Pulse return in $30 \mathrm{~s}$ & 13 \\
Additional arrest events & 3 \\
Died prior to discharge & 1 \\
Length of stay in hospital (days) & $17 \pm 35$ \\
& \\
Discharge destination & 17 \\
Home & 1 \\
Other hospital & 1 \\
Skilled nursing facility & 1 \\
Hospice & \\
\hline
\end{tabular}

talization, which is twice the reported survival rate of pediatric in-hospital arrest overall $(2,10)$.

Nearly a third of the CPAs that occurred in the PACU in this sample occurred in infants, which is similar to previous studies showing higher risk for CPA in infants $(1,2)$. Although some studies suggest that infants 
are less likely to survive CPA $(2,11)$, this study found that survival was associated with younger age in the PACU setting. Young age has been previously associated with lower mortality after CPA in noncardiac surgery (2) and in a pediatric intensive care setting that controlled for severity of illness (12). While surgical procedures were not recorded in the database, it could be speculated that the association between young age and greater survival in our sample is because the children likely underwent noncardiac surgery because those undergoing cardiac surgery generally bypass the PACU and go directly to the intensive care unit (ICU). Preexisting conditions were reported for more than $75 \%$ of the children in this sample of children experiencing CPA, with congenital heart disease observed far more frequently in this sample than in the general population, which is also consistent with prior studies of CPA in children (13). However, underlying conditions were not associated with survival in this small sample.

Respiratory issues were most frequently listed as causes for the CPA, which is consistent with the early reports of perioperative CPA $(14,15)$. Despite recently reported declines in CPA due to respiratory causes (1). this finding suggests an ongoing, albeit rare risk of early postoperative respiratory events in children. The cause of CPA events likely varies by phase of care as respiratory events have been shown to make up the majority of the causes reported for the postsurgical period $(1,16,17)$. Indeed, the Perioperative Cardiac Arrest registry reported that half of the CPAs in the postsurgical phase of care were due to respiratory causes, compared with $<30 \%$ due to cardiovascular causes. In this study, children with a cardiac/hemodynamic-related cause for CPA were less likely to survive the event compared with those with respiratory or mixed reasons, which is also consistent with previous literature (2).

More than half of the reported arrests occurred at mixed population facilities, and these children were less likely to survive the CPA event, despite being significantly older and having a similar prevalence of comorbidities compared with those at pediatric only hospitals. It could be speculated that differences in facilities, monitoring, response, or perhaps, provider expertise contributed to better outcomes in the pediatric facilities. However, the lack of detail regarding the nature of the cases in this study and the small number of deaths precluded the ability to adequately adjust this finding for the possible influence of these and other factors. Other studies have shown a decreased incidence of CPA in children cared for by pediatric compared with nonpediatric anesthesiologists (15); however, improved outcome following CPA based on pediatric hospital setting has previously only been demonstrated for children in inten- sive care setting (18) and for those undergoing sedation (19). This finding warrants further study, given the potential implications for children's outcomes.

Providers activated the emergency resuscitation team for only a third of CPA events in this sample - during only one of the weekend and none of the nighttime events - despite previous data suggesting the efficacy of such teams in reducing mortality (20). The use of the emergency resuscitation team was not significantly associated with survival in this sample, which may reflect early presence of the anesthesia team and/or early intervention, particularly given the finding that all CPA events were witnessed. Lastly, similar to earlier studies $(3,21)$, poor outcomes were associated with arrest on weekends and during the night. This finding may reflect the probable emergent nature of the anesthetic during these timeframes, which has been previously demonstrated $(1,9,22)$ or limited help and resources available on nights and weekends. Again, these data must be cautiously interpreted given that this is based on two nighttime arrests.

The voluntary, multicenter nature of the database used in this study poses the potential for selection and reporting biases, limiting the ability to generalize findings. In particular, bradycardic infants not receiving chest compressions will not have been captured. Additionally, the absence of PACU population information (i.e., denominator) precludes the ability to report an incidence of CPA or examine possible reporting biases. Likewise, the lack of perioperative data of interest (e.g., ASA physical status, surgery performed, anesthetic management, etc.) precludes examining certain risk factors of importance to anesthesia providers. The nature of data entry (i.e., check box format with a lack of narrative) and the absence of a root cause analysis approach make interpretation of the nature and cause of events difficult. For instance, the purported lack of intravenous access prearrest may indicate a deficiency in reporting, or, perhaps, a loss of or inability to obtain access, or even its early removal in preparation for discharge. Errors in reporting likely exist given that only one child appeared to have access pre-event in the nonsurvivor group whereas three were purportedly receiving vasoactive agents (Table 3). Furthermore, use of invasive airways prearrest and during arrest is difficult to interpret, given the lack of knowledge regarding the nature of the surgical procedures and planned admission status of the children (i.e., inpatient versus outpatient). These data must therefore be interpreted with caution. Lastly, the small sample size precluded the ability to conduct adjusted multivariate analyses of risk factors.

Compared with other reports of pediatric CPA, this study found a similar predominance of risk factors and 
underlying respiratory causes for arrest, with higher mortality following cardiac/hemodynamic causes. This study suggests several research and educational opportunities. Assuming that these findings are confirmed in larger samples amenable to multivariate analysis, training of PACU staff, and assurance of adequate resources should be directed to the recognition, prevention, and early management of respiratory arrest as the leading cause of PACU CPA. Further research is required to examine the cardiac/hemodynamic arrests. Appropriate clinical guidelines might direct these patients to bypass PACU in favor of direct ICU admission. Given the better survival rate for children in pediatric only facilities and during regular hospital hours, there may be opportunities to decrease mortality by, whenever possible, redirecting children to pediatric facilities and avoiding off-hours anesthesia, or ensuring adequate training and resources in mixed facilities. While survival from PACU CPA in this study is significantly better than pediatric CPA in other settings, these represent opportunities for improvement.

\section{Funding}

This study was supported by no grants or other funding.

\section{Conflicts of interest}

All authors report no conflicts of interest.

\section{References}

1 Bhananker S, Ramamoorthy C, Geiduschek $\mathrm{J}$ et al. Anesthesia-related cardiac arrest in children: update from the Pediatric Perioperative Cardiac Arrest Registry. Anesth Analg 2007; 105: 344-350.

2 Flick R, Sprung J, Harrison T et al. Perioperative cardiac arrests in children between 1988 and 2005 at a tertiary referral center: a study of 92,881 patients. Anesthesiology 2007; 106: 226-237.

3 Ahmed A, Ali M, Khan M et al. Perioperative cardiac arrests in children at a university teaching hospital of a developing country over 15 years. Pediatr Anesth 2009; 19: 581-586.

4 Peberdy M, Kaye W, Ornato J et al. Cardiopulmonary resuscitation of adults in the hospital: a report of 14720 cardiac arrests from the National Registry of Cardiopulmonary Resuscitation. Resuscitation 2003; 58: 297308.

5 Mhyre J, Ramachandran S, Kheterpal S et al. Delayed time to defibrillation after intraoperative and periprocedural cardiac arrest. Anesthesiology 2010; 113: 782-793.

6 Zaritsky A, Nadkarni V, Hazinski MF et al. Recommended guidelines for uniform reporting of pediatric advanced life support: the pediatric Utstein Style. A statement for healthcare professionals from a task force of the American Academy of Pediatrics, the American Heart Association, and the European Resuscitation Council. Writing Group. Circulation 1995; 92: 2006-2020.

7 Jacobs I, Nadkarni V, Bahr J et al. Cardiac arrest and cardiopulmonary resuscitation outcome reports: update and simplification of the Utstein templates for resuscitation registries: a statement for healthcare profes- sionals from a task force of the International Liaison Committee on Resuscitation (American Heart Association, European Resuscitation Council, Australian Resuscitation Council, New Zealand Resuscitation Council, Heart and Stroke Foundation of Canada, InterAmerican Heart Foundation, Resuscitation Councils of Southern Africa). Circulation 2004; 110: 3385-3397.

8 Samson R, Nadkarni V, Meaney P et al. Outcomes of in-hospital ventricular fibrillation in children. $N$ Engl J Med 2006; 354: 2328-2339.

9 Morray JP, Geiduschek JM, Ramamoorthy $\mathrm{C}$ et al. Anesthesia-related cardiac arrest in children: initial findings of the Pediatric Perioperative Cardiac Arrest (POCA) Registry. Anesthesiology 2000; 93: 6-14.

10 Nadkarni V, Larkin G, Peberdy M et al. First documented rhythm and clinical outcome from in-hospital cardiac arrest among children and adults. JAMA 2006; 295: 50-57.

11 Kawashima Y, Seo N, Morita K et al. Anesthesia-related mortality and morbidity in Japan (1999). J Anesth 2002; 16: 319-331.

12 Meaney P, Nadkarni V, Cook EF et al. Higher survival rates among younger patients after pediatric intensive care unit cardiac arrests. Pediatrics 2006; 118 : 2424-2433.

13 Ramamoorthy C, Haberkern C, Bhananker $\mathrm{S}$ et al. Anesthesia-related cardiac arrest in children with heart disease: data from the Pediatric Perioperative Cardiac Arrest (POCA) registry. Anesth Analg 2010; 110: 1376-1382.

14 Rackow H, Salanitre E, Green LT. Frequency of cardiac arrest associated with anes- thesia in infants and children. Pediatrics 1961; 28: 697-704.

15 Keenan RL, Shapiro JH, Dawson K. Frequency of anesthetic cardiac arrests in infants: effect of pediatric anesthesiologists. $J$ Clin Anesth 1991; 3: 433-437.

16 Cohen MM, Cameron CB, Duncan PG. Pediatric anesthesia morbidity and mortality in the perioperative period. Anesth Analg 1990; 70: 160-167.

17 McConachie IW, Day A, Morris P. Recovery from anaesthesia in children. Anaesthesia 1989; 44: 986-990.

18 Donoghue A, Nadkarni V, Elliott M et al. Effect of hospital characteristics on outcomes from pediatric cardiopulmonary resuscitation: a report from the national registry of cardiopulmonary resuscitation. Pediatrics 2006; 118: 995-1001.

19 Cote CJ, Notterman DA, Karl HW et al. Adverse sedation events in pediatrics: a critical incident analysis of contributing factors. Pediatrics 2000; 105: 805-814.

20 Tibballs J, Kinney S. Reduction of hospital mortality and of preventable cardiac arrest and death on introduction of a pediatric medical emergency team. Pediatr Crit Care Med 2009; 10: 306-312.

21 Sprung J, Warner M, Contreras M et al. Predictors of survival following cardiac arrest in patients undergoing noncardiac surgery: a study of 518,294 patients at a tertiary referral center. Anesthesiology 2003; 99: 259-269.

22 Braz L, Braz JRC, Módolo NSP et al. Perioperative cardiac arrest and its mortality in children. A 9-year survey in a Brazilian tertiary teaching hospital. Pediatr Anesth 2006; 16: $860-866$. 
Appendix 1 American Heart Association's Get With The Guidelines ${ }^{\circledR}$-Resuscitation (formerly the National Registry of Cardiopulmonary Resuscitation) Investigators

Mary E. Mancini, RN, PhD

Robert A. Berg, MD

Emilie Allen, RN, BSN

Elizabeth A. Hunt, MD

Vinay M. Nadkarni, MD

Mary Ann Peberdy, MD

Joseph P. Ornato, MD

Scott Braithwaite, MD

Graham Nichol, MD

Kathy Duncan, RN
Robert Faillace, MD

Robert Clark, MD

Heidi J. Dalton, MD

Peter C. Laussen, MB BS

Frank W. Moler, MD

Marilyn Morris, MD

Chris Parshuram, MD

Tia T. Raymond, MD

Arno L. Zaritsky, MD 